This item was submitted to Loughborough's Research Repository by the author.

Items in Figshare are protected by copyright, with all rights reserved, unless otherwise indicated.

\title{
Gap analysis of ontology mapping tools and techniques
}

PLEASE CITE THE PUBLISHED VERSION

https://doi.org/10.1007/978-1-84996-257-5_28

\section{PUBLISHER}

(c) Springer-Verlag

\section{VERSION}

AM (Accepted Manuscript)

\section{PUBLISHER STATEMENT}

This work is made available according to the conditions of the Creative Commons Attribution-NonCommercialNoDerivatives 4.0 International (CC BY-NC-ND 4.0) licence. Full details of this licence are available at: https://creativecommons.org/licenses/by-nc-nd/4.0/

\section{LICENCE}

CC BY-NC-ND 4.0

\section{REPOSITORY RECORD}

Anjum, Najam A., Jennifer A. Harding, R.I.M. Young, and Keith Case. 2019. "Gap Analysis of Ontology Mapping Tools and Techniques". figshare. https://hdl.handle.net/2134/27853. 


\title{
Gap Analysis of Ontology Mapping Tools and Techniques
}

\author{
Najam Anjum* , Jenny Harding ${ }^{*}$, Bob Young ${ }^{*}$ and Keith Case ${ }^{*}$ \\ Address Wolfson School of Mechanical and Manufacturing Engineering, Loughborough \\ University, Loughborough, LE11 3TU
}

\begin{abstract}
Mapping between ontologies provides a way to overcome any dissimilarities in the terminologies used in two ontologies. Some tools and techniques to map ontologies are available with some semi-automatic mapping capabilities. These tools are employed to join the similar concepts in two ontologies and overcome the possible mismatches. Several types of mismatches have been identified by researchers and certain overlaps can easily be seen in their description. Analysis of the mapping tools and techniques through a mismatches framework reveals that most of the tools and techniques just target the explication side of the concepts in ontologies and a very few of them opt for the conceptualization mismatches. Research therefore needs to be done in the area of detecting and overcoming conceptualization mismatches that may occur during the process of mapping.
\end{abstract}

Keywords: Ontology Mapping, Ontology Mismatches, Ontology Mapping Tools and Techniques

\section{Introduction}

Ontologies have proven to be very helpful in explicitly defining concepts along with their relations and attributes in a formalized way. The characteristic of ontologies being sharable requires the formulation of techniques to allow seamless knowledge transfer between them. This problem of interoperability can be resolved by mapping ontologies. The tools and techniques available for mapping ontologies, however, are not fully automatic and most parts of the mapping process require human involvement. In order to make these tools more reliable and automatic, the mismatches that exist in ontologies need to be studied carefully and the tools available for their detection and resolution are require analysis from the mismatches perspective. In this paper an effort has been made to review the ontology mismathces identified by researchers. A framework is then developed 
from this review and this is then used to analyze some of the available mapping tools and techniques. The results of this analysis are discussed afterwards.

\section{Mapping of Ontologies}

Mapping is the process in which for each concept in the source ontology a corresponding concept with similar semantics in the target ontology is found (Ehrig \& Staab, 2004). Typically a mapping process consists of three main stages. 1) Mapping discovery, 2)Mapping representation and 3)Mapping execution (Bruijn et al, 2006). Since there needs to be a similarity in the ontologies to be mapped, the mapping discovery stage corresponds to a search for this similarity. Once the similarities are detected a mapping plan is generated in the mapping representation stage and finally the mapping is executed.

Due to the heterogeneous nature of ontologies, the mapping process is subjec to mismatches in their components and building blocks. Being the specification of a conceptualization, an ontology is considered to consist of five components and sets of their definitions. In this scenario an ontology consists of a set of Class definitions, a set of Function definitions, a set of Relation definitions, a set of Instance definitions and a set of Axiom definitions (Visser et al, 1997). Differences in the way these five components are defined give ways in which ontologies can mismatch and these are now discussed.

\subsection{Ontology Mismatches}

Different types of mismatches have been defined by different authors. The most quoted classification is the one given by Visser et al (1997) who divided the semantic mismatches into two main types namely Conceptualization mismatches and Explication mismatches. Some other mismatches have also been identified. A brief overview of these mismatches is given below.

\subsubsection{Conceptualisation Mismatches}

These mismatches occur either due to a difference in the way conceptualisations are distinguished in two ontologies or the way they are related to each other in an ontology. Hence two different types of mismatches are Class mismatches and Relation mismatches.

Class Mismatch: This mismatch occurs due to the way classes in two ontologies are differentiated from each other. This mismatch can further be divided into two types namely a categorisation mismatch and aggregation level mismatch. A categorisation mismatch takes place when two similar classes in two ontologies contain different subclasses. An aggregation level mismatch arises when two ontologies define a similar class at different levels of abstraction.

Relation Mismatch: This mismatch happens due to the difference in relations and attributes of classes. Three further subdivisions of this type of mismatch are 
structure mismatch, attribute assignment mismatch and attribute type mismatch. Structure mismatch occurs when a conceptualisation is specified in two ontologies using a similar set of classes or subclasses but the structuring and relation setting is different. Attribute assignment mismatch occurs when two ontologies assign attributes to two similar classes differently. Attribute type mismatch comes into play when two ontologies in their classes contain similar instances but these instances differ in the way they are defined.

\subsubsection{Explication Mismatches}

Explication mismatches are due to the difference in the way conceptualisations are defined in an ontology. The definitions of classes, relations and instances are considered to be a 3-tuple of terms, definiens and concepts i.e. Def $=\langle T, D, C\rangle$ (Visser et al, 1997). An explication mismatch can arise when any of these three components of the 3-tuple in two ontologies are different in some way. The relation between the terms, definiens and concepts is that definiens use terms to define a concept. For example, the definition of a Pen can be 'a writing device' or it can be 'a hollow cylinder filled with ink'. Both of these definitions attempt to describe the concept of a pen but they use different definiens and different terms. In the first one the definiens target the application of the pen while in the second one the structure of a pen is made the basis of its description. With these differences in terms, definiens and concepts, there can be six combinations of explication mismatches in ontologies. These are: concept mismatches (CMismatches), definiens mismatches (D-Mismatches), term mismatches (TMismatches), concept and definien mismatches (CD-Mismatches), concept and term mismatches (CT-Mismatches) and finally term and definien mismatches (TDMismatches). These mismatches are discussed below.

\subsubsection{Concept Description Mismatches:}

Named as Modelling Convention mismatch by Chalupsky (2000), this type of mismatch comes under the category of Class Mismatch of Visser et al. This specific type, however, is not identified by them and therefore becomes an additional type of Class Mismatch. Concept description mismatch occurs when a concept is defined using different sub or super-classes. For example, Chalupsky (2000) states that to distinguish between tracked and wheeled vehicles, a choice one way is to make two subclasses of Vehicle as Tracked-Vehicle and WheeledVehicle. Alternatively, an attribute of Wheeled can be defined with a relation of Traction-type.

\subsubsection{Model Coverage and Granularity Mismatch:}

This is another type of the class mismatch of Visser et al defined by Klien (2001) and Chaplusky (2000) as Model Coverage and Granularity mismatch. As the name suggests, this mismatch occurs when two ontologies define the same concept with different levels of granularity. For example, a list of names can come under a class Persons or to make it more detailed, the class Person can further be divided into Male and Female. This mismatch appears to be similar to the aggregation level 
mismatch of Visser et al (1997) but this similarity is not recognized by Klien (2001) and Chalupsky (2000).

\subsubsection{Single vs. Multi-Valued Property}

This is first of the three mismatches Qadir and colleagues (2007) claim to be different from the mismatches previously identified by other authors. This mismatch occurs when a data-type or object property is represented in the same class but take different number of values in two ontologies. The authors give an example of a class named Bank_Account. In the ontology of one bank, this class might take just one value because that bank doesn't allow its clients to have more than one account but in another, the class with same name might allow multiple values (i.e. to represent several different accounts) according to its policy.

\subsubsection{Unique vs. Non-Unique Valued Property}

This mismatch occurs when in one ontology a property can hold only one value that uniquely determines the subject, while in another ontology there can be multiple values but they cannot identify the subject uniquely (Qadir et al, 2007). Again quoting an example from the authors which explains the situation where in one ontology of a university, a student is identified by a unique rank number which is recognized by all departments while in another ontology the university requires multiple ranks corresponding to different departments and none of them individually determines the student uniquely.

\subsubsection{Alignment Conflict among Disjoint Relations}

A mismatch occurring when a disjoint relation in one ontology is not valid in the other. For example a class Student can be declared as disjoint with the class Employee in one ontology while in another, a student is also allowed to be an employee of an institution (Qadir et al, 2007).

\subsection{The Mismatches Framework}

Table 1 shows the framework formed by accumulating the possible ontological mismatches as described in the previous section. These mismatches are divided into two categories. The main list of semantic mismatches in relation to which all the other mismatches are analyzed is from Visser et al (1997). Their work is the most quoted one in the mismatches literature. Mismatches explained by other authors mostly overlap with those described by Visser and colleagues. For example categorization and aggregation level mismatches of Visser et al are similar to the scope differences of Wiederhold (1994) and scope mismatch of Klien (2001) and Qadir et al (2007). Similarly, the concept and definiens mismatches of Visser et al have a counterpart in the attribute scope mismatch of Wiederhold (1994) and homonym terms mismatch of Klien (2001). On the explication mismatch side, the concept and definiens mismatch of Visser et al (1997) has equivalents in Wiederhold (1994) and Klien (2001) with the names of Attribute Scopes and Homonym Terms mismatch respectively. Similarly, the Term mismatch and 
Table 1: Comparison of Identified Ontology Mismatches

\begin{tabular}{|c|c|c|c|c|c|c|c|}
\hline \multicolumn{2}{|c|}{$\begin{array}{l}\text { Mismatch } \\
\text { Category }\end{array}$} & \multirow{2}{*}{$\begin{array}{l}\text { Visser et al (1997) } \\
\text { Categorization Mm }\end{array}$} & \multirow{3}{*}{$\begin{array}{l}\begin{array}{l}\text { Wiederhold } \\
\text { (1994) }\end{array} \\
\text { Scope } \\
\text { Differences }\end{array}$} & \multirow{3}{*}{$\begin{array}{l}\text { Klien (2001) } \\
\text { Scope Mm }\end{array}$} & \multirow[t]{3}{*}{ Chaplusky (2000) } & \multirow{3}{*}{$\begin{array}{l}\text { Qadir et al (2007) } \\
\text { Scope Mm }\end{array}$} & \multirow{2}{*}{$\begin{array}{l}\text { Cummulative Mismatches } \\
\text { Categorization Mm }\end{array}$} \\
\hline \multirow{10}{*}{ 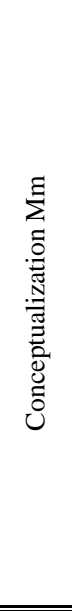 } & \multirow{6}{*}{$\sum_{\substack{\infty \\
0 \\
\Xi \\
U}}$} & & & & & & \\
\hline & & Aggregation-level Mm & & & & & Aggregation-level Mm \\
\hline & & & & Concept Description & Modelling Conventions & & Concept Description Mm \\
\hline & & & & $\begin{array}{l}\text { Model Coverage and } \\
\text { Granularity Mm }\end{array}$ & $\begin{array}{l}\text { Model Coverage and } \\
\text { Granularity Mm }\end{array}$ & & Coverage $\mathrm{Mm}$ \\
\hline & & & & & & Single vs Multi valued property & $\begin{array}{l}\begin{array}{l}\text { Single vs Multi valued } \\
\text { property }\end{array} \\
\end{array}$ \\
\hline & & & & & & $\begin{array}{l}\text { Unique vs Non-unique valued } \\
\text { property }\end{array}$ & $\begin{array}{l}\text { Unique vs Non-unique } \\
\text { valued property }\end{array}$ \\
\hline & \multirow{4}{*}{ 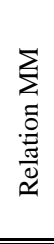 } & Structure Mm & & & & & Structure Mm \\
\hline & & $\begin{array}{l}\text { Attribute-assignment } \\
\text { Mm }\end{array}$ & & & & & Attribute-assignment Mm \\
\hline & & Attribute-type Mm & & & & & Attribute-type Mm \\
\hline & & & & & & $\begin{array}{ll}\begin{array}{l}\text { Alignment conflict } \\
\text { disjoint relations }\end{array} & \text { among } \\
\end{array}$ & $\begin{array}{l}\text { Alignment conflict among } \\
\text { disjoint relations }\end{array}$ \\
\hline \multirow{6}{*}{ 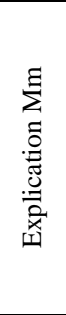 } & \multicolumn{2}{|c|}{ Concept \& Term Mm } & & & & & Concept \& Term Mm \\
\hline & \multicolumn{2}{|c|}{ Concept \& Definiens Mm } & Attribute Scopes & Homonym Terms Mm & & & Concept \& Definiens Mm \\
\hline & \multicolumn{2}{|c|}{ Concept Mm } & & & & & Concept Mm \\
\hline & \multicolumn{2}{|c|}{ Term \& Definiens Mm } & & Synonym Terms Mm & & & Term \& Definiens Mm \\
\hline & \multicolumn{2}{|c|}{ Term Mm } & $\begin{array}{l}\text { Naming } \\
\text { Differences }\end{array}$ & & & & Term Mm \\
\hline & \multicolumn{2}{|c|}{ Definiens Mm } & $\begin{array}{l}\text { Encoding } \\
\text { Differences }\end{array}$ & Encoding Mm & & & Definiens Mm \\
\hline
\end{tabular}


Definiens mismatches of Visser et al (1997) are referred to as Naming Differences and Encoding Differences respectively in Wiederhold (1994).

\section{Mapping Tools and Techniques}

Table 2 lists some of the main techniques used to map ontologies. These techniques include frameworks like MAFRA, OIS, IFF and mapping methods and tools like GLUE, FCA Merge, ONION. These techniques are analyzed for the similarity measures they take to align ontologies and the way they verify the connections made between the mapped ontologies. For the purpose of brevity, a description of these techniques is not included here. The summary of the similarity and verification parameters that these techniques use can be seen in Table 2 .

Table 2: Ontology Mapping Techniques

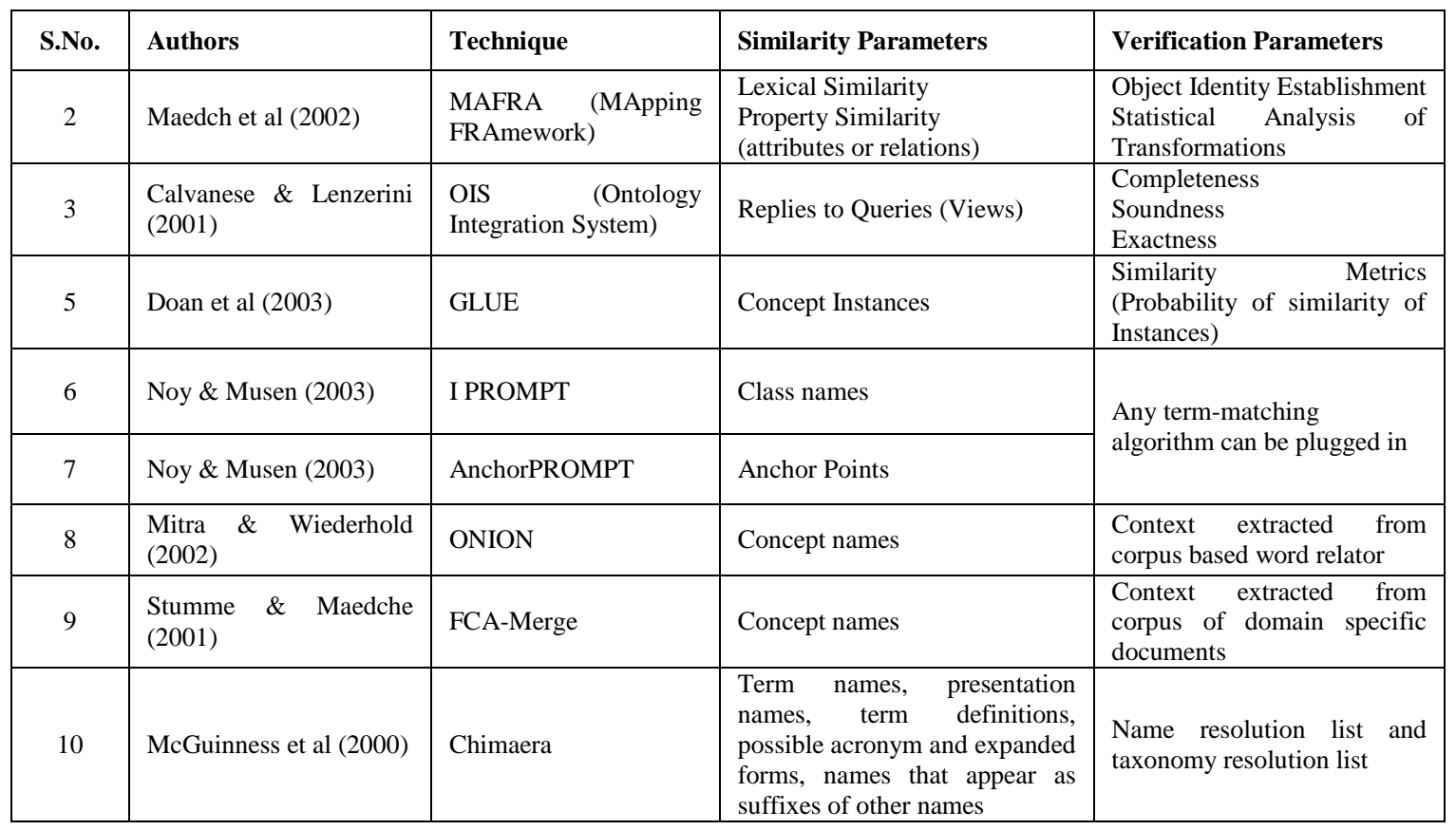

\section{Analysis}

Table 3 uses the mismatches framework developed from the review of typical ontological mismatches. The matrix formed here helps in analyzing the available 
Table 3: Analysis of Mapping Tools and Techniques from the Mismatches Point of View

\begin{tabular}{|c|c|c|c|c|c|c|c|c|c|c|c|c|c|c|c|c|c|}
\hline \multirow{2}{*}{\multicolumn{2}{|c|}{ Semantic Mismatches }} & \multicolumn{2}{|c|}{ MAFRA } & \multicolumn{2}{|c|}{ PROMPT } & \multicolumn{2}{|c|}{$\begin{array}{l}\text { Anchor- } \\
\text { PROMPT }\end{array}$} & \multicolumn{2}{|c|}{ GLUE } & \multicolumn{2}{|l|}{ QOM } & \multicolumn{2}{|c|}{ ONION } & \multicolumn{2}{|c|}{ FCA-Merge } & \multicolumn{2}{|c|}{ Chimera } \\
\hline & & Detection & $\begin{array}{l}\text { Resolution } \\
\text { Pesolin }\end{array}$ & $\begin{array}{l}\text { Detection } \\
\end{array}$ & $\begin{array}{l}\text { Resolution } \\
\text { P }\end{array}$ & 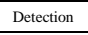 & 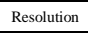 & \begin{tabular}{l|l|l|c|} 
Detction \\
\end{tabular} & $\begin{array}{l}\text { Resolution } \\
\text { R }\end{array}$ & Detection & $\begin{array}{l}\text { Resolution } \\
\text { P }\end{array}$ & $\begin{array}{l}\text { Detection } \\
\end{array}$ & $\begin{array}{l}\text { Resolution } \\
\text { P }\end{array}$ & $\begin{array}{l}\text { Detection } \\
\text { Detect }\end{array}$ & $\begin{array}{l}\text { Resolution } \\
\text { Patolon }\end{array}$ & 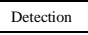 & $\begin{array}{l}\text { Resolution } \\
\text { R }\end{array}$ \\
\hline \multirow{10}{*}{ 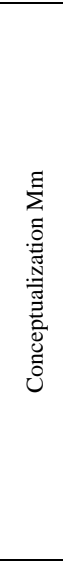 } & Categorization Mm & & & & & M & $\mathrm{U}$ & & & M & & & & & & M & \\
\hline & Aggregation-level Mm & & & & & A & $\mathrm{U}$ & & & M & & & & & & M & \\
\hline & Concept Description Mm & & & & & & & & & M & & & & & & $\mathrm{M}$ & \\
\hline & Coverage $\mathrm{Mm}$ & & & & & & & & & M & & & & & & $\mathrm{M}$ & \\
\hline & $\begin{array}{l}\begin{array}{l}\text { Single vs Multi valued } \\
\text { property }\end{array} \\
\end{array}$ & & & & & & & M & & M & & & & & & M & \\
\hline & $\begin{array}{l}\text { Unique vs Non-unique } \\
\text { valued property }\end{array}$ & & & & & & & M & & M & & & & & & M & \\
\hline & Structure Mm & & & & & & & & & M & & & & & & M & \\
\hline & Attribute-assignment Mm & & & & & & & & & M & & & & & & M & \\
\hline & Attribute-type Mm & & & & & & & & & M & & & & & & M & \\
\hline & $\begin{array}{l}\text { Alignment conflict among } \\
\text { disjoint relations } \\
\end{array}$ & & & & & & & & & M & & & & & & M & \\
\hline \multirow{6}{*}{ 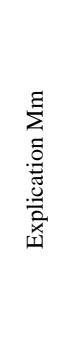 } & Concept \& Term Mm & & & & & & & & & M & & & & & & M & \\
\hline & $\begin{array}{l}\text { Concept \& Definiens Mm } \\
\text { (Homonyms) }\end{array}$ & & & & & & & & & M & & & & & & M & \\
\hline & Concept Mm & & & & & & & & & M & & & & & & M & \\
\hline & $\begin{array}{l}\text { Term \& Definiens Mm } \\
\text { (Synonyms) }\end{array}$ & M & & M & $\mathrm{U}$ & A & $\mathrm{U}$ & A & $\mathrm{U}$ & A & $\mathrm{U}$ & M & $\mathrm{U}$ & A & $\mathrm{U}$ & A & $\mathrm{U}$ \\
\hline & Term Mm & M & & M & $\mathrm{U}$ & $\mathrm{A}$ & $\mathrm{U}$ & $\mathrm{A}$ & $\mathrm{U}$ & A & $\mathrm{U}$ & M & $\mathrm{U}$ & A & $\mathrm{U}$ & $\mathrm{A}$ & $\mathrm{U}$ \\
\hline & Definiens Mm & M & & M & $\mathrm{U}$ & A & $\mathrm{U}$ & A & $\mathrm{U}$ & A & $\mathrm{U}$ & M & $\mathrm{U}$ & A & $\mathrm{U}$ & A & $\mathrm{U}$ \\
\hline
\end{tabular}


tools and techniques from the mismatches point of view. Three symbols are used here to denote the capability of a particular method to detect and resolve a mismatch as done by Klien (2001). ' $A$ ' stands for automatic and represents a capability of automatically detecting or resolving a mismatch. ' $U$ ' stands for user and symbolizes the suggestions a tool offers to the user to solve a particular mismatch, and ' $M$ ' denotes the mechanism provided to the user, by a tool or technique, to detect or resolve a mismatch. Before any results are obtained from this analysis, it is necessary to clarify that the tools and techniques are designed to find out similarities while the mismatches literature stresses the dissimilarities that are present among ontologies. Hence, the fields filled in Table 3 indicate that a certain tool or technique overcomes a particular mismatch in one ontology by connecting a differently placed or named concept to a corresponding concept in another ontology.

A quick glimpse of this table reveals some empty fields representing a lack of available features in tools and techniques to detect and resolve conceptualization mismatches. Most of the tools and techniques provide a mechanism to the user to detect and resolve mismatches. It can be seen from table 3 that QOM (Quick Ontology Mapping) and Chimaera have a mechanism for the users to detect the conceptualization mismatches. This is because in QOM the breadth of scope of similarity measure allows this technique to cover all of the mismatches to be detected. In Chimaera, however, it is its detailed and user friendly interface that helps the user to manually detect any kind of mismatches. This on one hand shows that the available tools and techniques need to be made more automatic and on the other it indicates that these tools should be modified to target conceptualization mismatches. It is also clear from table 2 that the available tools and techniques mainly focus on finding the similarities rather than dissimilarities between the concepts in two ontologies and then establishing correspondences. So the main steps involved in every technique are:

1- Scanning ontologies for similar concepts,

2- Authenticating the similarity through different algorithms and tools,

3- Establishing correspondences.

The second step is the one which deals with the verification of knowledge in shared ontologies and it is here that the research so far is mainly directed towards the explication side of terminologies and concepts. The conceptualization side of the interpretation of terms and concepts is virtually void of any significant work Table 3 shows that only AnchorPROMPT provides an automatic detection of one of the conceptualization type of similarities and also suggests the possible correspondence that can be established between specific concepts in the ontologies to be mapped. The other two tools QOM and Chimaera just provide information about the structure of ontologies so that it becomes easier for the user to detect some conceptualization similarity.

The gap identified here suggests thatresearch is required to find ways through which different conceptualization mismatches can be detected and resolved in 
order to give accuracy to the process of mapping and thus verifying the knowledge being shared.

\section{References}

Bruijn, J. de., Ehrig, M., Feier, C., Marin-Recuerda, F., Scharffe, F. and Wieten, M., 2006, "Ontology Mediation, Merging and Aligning”, In Davies J, Studer R, Warren P (eds), Semantic Web Technologies: Trends and Research in Ontologybased Systems, Wiley, UK, 2006

Calvanese, D. and Lenzerini, M., 2001. Ontology of integration and integration of ontologies, In Proceedings of the 2001 Description Logic Workshop (DL, 2001, pp10-19.

Chaplusky, H, 2000, "OntoMorph: A translation system for symbolic knowledge", Proc. 17th Intl. Conf. on Principles of Knowledge Representation and Reasoning (KR'2000)

Doan, ,Anhai, Madhavan, ,Jayant, Domingos, ,Pedro and Halevy, ,Alon, 2002. Learning to map between ontologies on the semantic web, $W W W^{\prime}$ '02: Proceedings of the 11th international conference on World Wide Web, 2002, ACM pp662-673.

Ehrig, M. and Staab, S., 2004, “QOM - Quick Ontology Mapping”, pp. 683-697.

Klien, M, 2001, "Combining and relating ontologies: an analysis of problems and solutions", Workshop on Ontologies and Information Sharing, IJCAI'01

Maedche, ,Alexander, Motik, ,Boris, Silva, ,Nuno and Volz, ,Raphael, 2002, "MAFRA - A MApping FRAmework for Distributed Ontologies", EKAW '02: Proceedings of the 13th International Conference on Knowledge Engineering and Knowledge Management. Ontologies and the Semantic Web, 2002, SpringerVerlag pp235-250.

McGuiness, D.L., Fikes, R., RICE, J. and Wilder, S., 2000, “An environment for merging and testing large ontologies", Proc. 17th Intl. Conf. on Principles of Knowledge Representation and Reasoning (KR'2000)

Mitra, P. and Wiederhold, G., 2002, "Resolving terminological heterogeneity in ontologies", Workshop on Ontologies and Semantic Interoperability at the 15th European Conference on Artificial Intelligence (ECAI-2002)

Noy, N. F. and Musen, M. A., 2003, "The PROMPT suite: interactive tools for ontology merging and mapping", International Journal of Human-Computer Studies, Vol 59 , No. 6,pp 983 - 1024 
Qadir, M. A., Fahad, M. and Noshairwan, M. W., 2007, "On conceptualization mismatches between ontologies", IEEE International Conference on Granular Compution (2007)

Stumme, G. and Maedche, A., 2001, “Ontology margining for federated ontologies on the semantic web", In Proceedings of the International Workshop for Foundations of Models for Information Integration (FMII-2001), pp 413-418

Visser, P.R.S., Jones, D.M., Bench-Capon, T.J.M. and Shave, M.J.R.,1997, An Analysis of Ontology Mismatches; Heterogeneity versus Interoperability In AAAI1997 Spring Symposium on Ontological Engineering, Stanford, USA.

Wiederhold, G, 1994, “An algebra for ontology composition”, In Proceedings of 1994 Monterey Workshop on Formal Methods, pp 56-61 